EPJ Web of Conferences 113,06002 (2016)

DOI: $10.1051 /$ epjconf/201611306002

(C) Owned by the authors, published by EDP Sciences, 2016

\title{
Towards an ab initio description of light-nuclei radiative captures
}

\author{
Jérémy Dohet-Eraly ${ }^{1, a}$, Petr Navrátil ${ }^{1}$, Sofia Quaglioni ${ }^{2}$, Wataru Horiuchi ${ }^{3}$, and Guillaume Hupin ${ }^{2}$ \\ ${ }^{1}$ TRIUMF, 4004 Wesbrook Mall, Vancouver BC V6T 2A3, Canada \\ ${ }^{2}$ Lawrence Livermore National Laboratory, P.O. Box 808, L-414, Livermore, California 94551, USA \\ ${ }^{3}$ Department of Physics, Hokkaido University, Sapporo 060-0810, Japan
}

\begin{abstract}
The ${ }^{3} \mathrm{He}(\alpha, \gamma)^{7} \mathrm{Be}$ and ${ }^{3} \mathrm{H}(\alpha, \gamma)^{7} \mathrm{Li}$ astrophysical $S$ factors are evaluated at low collision energies (less than $2.5 \mathrm{MeV}$ in the centre-of-mass frame) within the no-core shell model with continuum using a renormalized chiral nucleon-nucleon interaction.
\end{abstract}

\section{Introduction}

Radiative-capture processes play an important role in stellar nucleosynthesis. Of particular interest is the ${ }^{3} \mathrm{He}(\alpha, \gamma)^{7} \mathrm{Be}$ cross section or astrophysical $S$ factor at collision energies about $20 \mathrm{keV}$ in the centre-of-mass (c.m.) frame, which is essential to evaluate the fraction of pp-chain terminations resulting in ${ }^{7} \mathrm{Be}$ neutrinos [1,2]. The study of the ${ }^{3} \mathrm{He}(\alpha, \gamma)^{7} \mathrm{Be}$ reaction at low energies, complemented by the study of its mirror reaction, the ${ }^{3} \mathrm{H}(\alpha, \gamma)^{7} \mathrm{Li}$ radiative capture, is also needed to evaluate the primordial ${ }^{7} \mathrm{Li}$ abundance in the universe [3]. In spite of the interest in these capture reactions, measurements at astrophysical energies have not been possible because at such low energies, the capture cross sections are very small due to the Coulomb repulsion between the colliding nuclei. Theoretical models (or extrapolations) are thus needed to provide the ${ }^{3} \mathrm{He}(\alpha, \gamma){ }^{7} \mathrm{Be}$ and ${ }^{3} \mathrm{H}(\alpha, \gamma){ }^{7} \mathrm{Li}$ capture cross sections at solar energies.

The ${ }^{3} \mathrm{He}(\alpha, \gamma){ }^{7} \mathrm{Be}$ astrophysical $S$ factor has been measured for collision energies between $\sim 0.1$ and $3 \mathrm{MeV}$ in the last decade [4-10] with significantly increased accuracy with respect to the previous experiments [1]. These recent experimental data provide a useful ground test to check the accuracy of theoretical models, which are then used to predict the astrophysical $S$ factor at lower energies. In contrast, the ${ }^{3} \mathrm{H}(\alpha, \gamma)^{7} \mathrm{Li}$ astrophysical $S$ factor is known less accurately and has been only measured between $\sim 50 \mathrm{keV}$ and $1.2 \mathrm{MeV}[11,12]$.

Many theoretical models have been developed to describe these radiative capture processes: from simple external-capture models [13] to much more complicated microscopic approaches [3, 14-17]. However, no ab initio approach, i.e. a microscopic approach based on a realistic inter-nucleon interaction able to describe accurately bound states and continuum states in a consistent framework, has been developed, yet. In this proceeding, we present an application of the no-core shell model with continuum (NCSMC) approach [18], using a renormalized chiral nucleon-nucleon (NN) interaction, to the description of the ${ }^{3} \mathrm{He}(\alpha, \gamma){ }^{7} \mathrm{Be}$ and ${ }^{3} \mathrm{H}(\alpha, \gamma){ }^{7} \mathrm{Li}$ radiative-capture processes. This work is a first step towards a complete $a b$ initio description of these radiative-capture reactions.

\footnotetext{
ae-mail: jdoheter@triumf.ca
} 


\section{No-core shell model with continuum approach}

The ${ }^{3} \mathrm{He}(\alpha, \gamma){ }^{7} \mathrm{Be}$ and ${ }^{3} \mathrm{H}(\alpha, \gamma){ }^{7} \mathrm{Li}$ astrophysical $S$ factors are calculated from the matrix elements of the electromagnetic transition multipole operators between initial scattering states and final bound states [19]. The initial and final states are obtained with the NCSMC approach [18], as solutions of a microscopic Schrödinger equation based on a realistic nucleon-nucleon interaction. In this approach, each partial wave function $\left|\psi^{J^{\pi} T}\right\rangle$ of total angular momentum $J$, parity $\pi$, and isospin $T$ is described by a combination of $\alpha+{ }^{3} \mathrm{He}$ or $\alpha+{ }^{3} \mathrm{H}$ cluster states and eigenstates of the compound ${ }^{7} \mathrm{Be}$ or ${ }^{7} \mathrm{Li}$ systems obtained with the no-core shell model (NCSM) [20]. The latter eigenstates, denoted by $\left.{ }^{7} \mathrm{Be} \lambda J^{\pi} T\right\rangle$ or $\left.\left.\right|^{7} \mathrm{Li} \lambda J^{\pi} T\right\rangle$ with $\lambda$ the energy label, are fully antisymmetric and translational-invariant. They are built from linear combinations of products of harmonic oscillator (HO) functions with frequency $\Omega$ and up to $N_{\max }$ HO quanta above the lowest configuration. The cluster states, denoted by $\left|\Phi_{v r}^{J^{\pi} T}\right\rangle$, are also translational-invariant. They are built from the NCSM states of ${ }^{4} \mathrm{He}$ and ${ }^{3} \mathrm{He}$ or ${ }^{3} \mathrm{H}$ by means of the resonating group method (RGM) [21]. For the ${ }^{3} \mathrm{He}(\alpha, \gamma)^{7} \mathrm{Be}$ study, they are given by

$$
\left.\left.\left|\Phi_{v r}^{J^{\pi} T}\right\rangle=\left[\left.\left[{ }^{4} \operatorname{He} \lambda_{\alpha} J_{\alpha}^{\pi_{\alpha}} T_{\alpha}\right\rangle\right|^{3} \mathrm{He} \lambda_{\mathrm{h}} J_{\mathrm{h}}^{\pi_{\mathrm{h}}} T_{\mathrm{h}}\right\rangle\right]^{(s T)} Y_{\ell}\left(\hat{r}_{\alpha \mathrm{h}}\right)\right]^{\left(J^{\pi} T\right)} \frac{\delta\left(r-r_{\alpha \mathrm{h}}\right)}{r r_{\alpha \mathrm{h}}},
$$

where $\left|{ }^{4} \mathrm{He} \lambda_{\alpha} J_{\alpha}^{\pi_{\alpha}} T_{\alpha}\right\rangle$ is a NCSM state of ${ }^{4} \mathrm{He}$ with $\lambda_{\alpha} J_{\alpha} \pi_{\alpha} T_{\alpha}$ standing for the energy label, total angular momentum, parity, and isospin associated with $\left.{ }^{4} \mathrm{He},{ }^{3} \mathrm{He} \lambda_{\mathrm{h}} J_{\mathrm{h}}^{\pi_{\mathrm{h}}} T_{\mathrm{h}}\right\rangle$ is a NCSM state of ${ }^{3} \mathrm{He}$ with similar definitions of the quantum numbers, $s$ is the channel spin, $\boldsymbol{r}_{\alpha \mathrm{h}}$ is the relative coordinate between the centres of mass of ${ }^{4} \mathrm{He}$ and ${ }^{3} \mathrm{He}, \ell$ is the relative orbital angular momentum between the clusters, and $v$ is a collective index for all relevant quantum numbers. An operator $\hat{\mathcal{A}}_{\alpha \mathrm{h}}$ is applied to enforce the antisymmetrization between the nucleons belonging to ${ }^{4} \mathrm{He}$ and those belonging to ${ }^{3} \mathrm{He}$. As an example, for the ${ }^{3} \mathrm{He}(\alpha, \gamma)^{7} \mathrm{Be}$ study the NCSMC partial wave can be written as

$$
\left.\left|\psi^{J^{\pi} T}\right\rangle=\left.\sum_{\lambda} c_{\lambda}^{J^{\pi} T}\right|^{7} \operatorname{Be} \lambda J^{\pi} T\right\rangle+\sum_{v} \int d r r^{2} \frac{\gamma_{v}^{J^{\pi} T}(r)}{r} \hat{\mathcal{A}}_{\alpha \mathrm{h}}\left|\Phi_{v r}^{J^{\pi} T}\right\rangle .
$$

The $c_{\lambda}^{J^{\pi} T}$ coefficients and the $\gamma_{v}^{J^{\pi} T}$ functions are determined by inserting the ansatz (2) in a variational form of the seven-nucleon Schrödinger equation. The proper asymptotic behaviour of a bound state or a scattering state is imposed by using the microscopic $R$-matrix method (MRM) [22, 23]. From the MRM, the asymptotic normalization coefficients for bound states and the collision matrix for scattering states (or, equivalently, the phase shift for one-open-channel studies) are determined.

\section{$\left.3{ }^{3} \mathrm{He}(\alpha, \gamma)\right)^{7} \mathrm{Be}$ and ${ }^{3} \mathrm{H}(\alpha, \gamma){ }^{7} \mathrm{Li}$ astrophysical $S$ factors}

A chiral $\mathrm{N}^{3} \mathrm{LO} \mathrm{NN}$ interaction [24] softened via the similarity-renormalization-group (SRG) method [25-27], which reduces the influence of momenta higher than $\hbar \Lambda$, is considered. The SRG transformation induces three-body forces even if the initial interaction is limited to two-body terms. For computational reasons, in this calculation, we restrict ourselves to the two-body part of the renormalized interaction. We adjust the SRG parameter $\left(\Lambda=2.15 \mathrm{fm}^{-1}\right.$ is used) to approximately reproduce the separation energies of the ${ }^{7} \mathrm{Be}$ and ${ }^{7} \mathrm{Li}$ bound states for the considered model space, which is essential to get the correct tail of the wave function and hence the astrophysical $S$ factors. This adjustement is required because three-nucleon forces are neglected and because the largest accessible model space is still too small to get converged results. Partial waves with $J^{\pi}=\left\{1 / 2^{+}, 3 / 2^{+}, 5 / 2^{+}, 1 / 2^{-}, 3 / 2^{-}\right\}$ and isospin $T=1 / 2$ are considered. The HO frequency is set to $\Omega=20 \mathrm{MeV} / \hbar$ for both NCSM and NCSM/RGM states. Nine ${ }^{7} \mathrm{Be}$ or ${ }^{7} \mathrm{Li}$ NCSM states are included in expansion (2): one in partial wave 

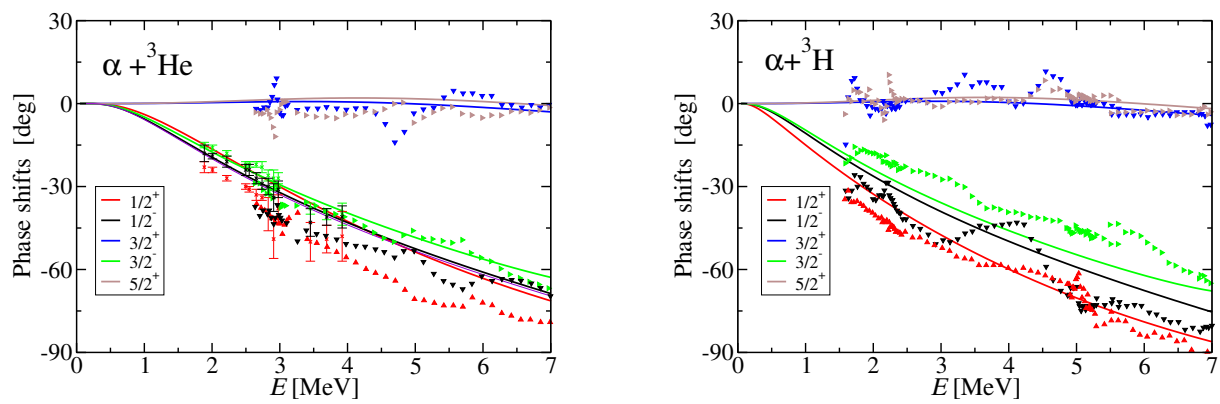

Figure 1. The $\alpha+{ }^{3} \mathrm{He}$ and $\alpha+{ }^{3} \mathrm{H}$ elastic phase shifts, obtained from the NCSMC and from experiments [28, 29]. See the text for model details.
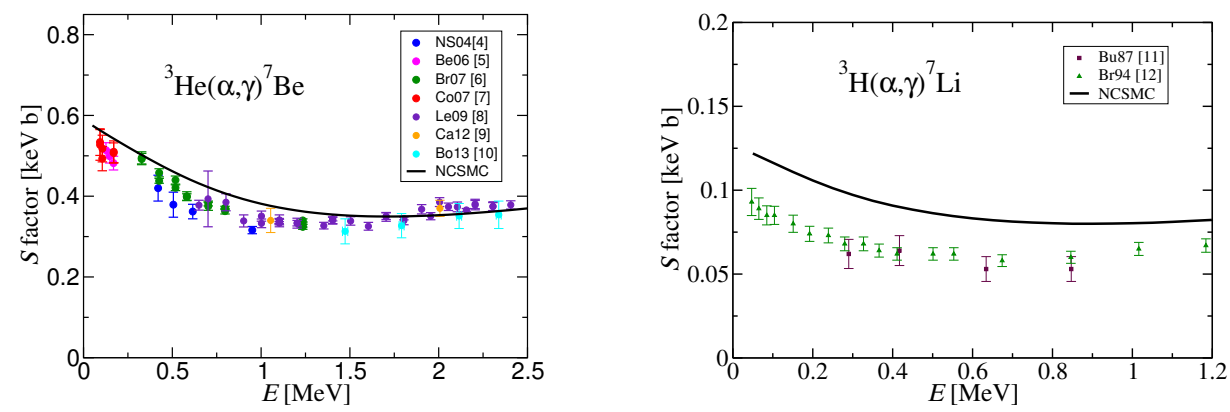

Figure 2. The ${ }^{3} \mathrm{He}(\alpha, \gamma)^{7} \mathrm{Be}$ and ${ }^{3} \mathrm{H}(\alpha, \gamma)^{7} \mathrm{Li}$ astrophysical $S$ factors obtained from the NCSMC and from experiments [4-12]. See the text for model details.

$J^{\pi}=5 / 2^{+}$and two in the other partial waves. Positive-parity states are obtained with $N_{\max }=11$ and negative-parity states with $N_{\max }=10$. The NCSM/RGM states are obtained by coupling the NCSM ground state of ${ }^{4} \mathrm{He}$, characterized by $\left(J^{\pi} T\right)=\left(0^{+} 0\right)$, with the $\left(1 / 2^{+} 1 / 2\right) \mathrm{NCSM}$ ground state of ${ }^{3} \mathrm{He}$ or ${ }^{3} \mathrm{H}$. Both are obtained with $N_{\max }=12$.

The computed $\alpha+{ }^{3} \mathrm{He}$ and $\alpha+{ }^{3} \mathrm{H}$ elastic phase shifts for relative collision energies $(E)$ up to $7 \mathrm{MeV}$ are compared with experiment $[28,29]$ in Fig. 1 . The agreement is not perfect, in particular for the $1 / 2^{+}$wave. However, the accuracy of the experimental data is quite poor, making a quantitative comparison difficult. The ${ }^{3} \mathrm{He}(\alpha, \gamma)^{7} \mathrm{Be}$ astrophysical $S$ factors for collision energies up to $2.5 \mathrm{MeV}$ and the ${ }^{3} \mathrm{H}(\alpha, \gamma)^{7} \mathrm{Li}$ astrophysical $S$ factors for collision energies up to $1.2 \mathrm{MeV}$ are compared, in Fig. 2, with the most recent and accurate experimental data [4-12]. For these ranges of energies, the $E 1$ transitions are dominant and thus, we neglect the other transitions. Qualitatively, the experimental ${ }^{3} \mathrm{He}(\alpha, \gamma)^{7} \mathrm{Be}$ astrophysical $S$ factor is rather well reproduced by the NCSMC approach but the ${ }^{3} \mathrm{H}(\alpha, \gamma){ }^{7} \mathrm{Li}$ astrophysical $S$ factor is overestimated. A similar mismatch was obtained by Neff with a microscopic appoach based on a NN realistic interaction [17]. A quantitative comparison requires the inclusion of three-body forces. By including them, we expect a reproduction of the experimental ${ }^{7} \mathrm{Be}$ and ${ }^{7} \mathrm{Li}$ separation energies without fine-tuning the SRG parameter $\Lambda$, involved in the renormalization of the interaction, and a smaller $\Lambda$-dependence of the results. Then, a smaller $\Lambda$ value could be considered, which would lead to converged results with a smaller model space. Overall, the predictive power of the approach would be improved. 


\section{Acknowledgements}

TRIUMF receives funding via a contribution through the National Research Council Canada. This work was supported in part by NSERC under Grant No. 401945-2011, by LLNL under Contract DE-AC52-07NA27344, by the U.S. Department of Energy, Office of Science, Office of Nuclear Physics, under Work Proposal Number SCW1158, and by JSPS KAKENHI Grant Numbers 25800121 and 15K05072. W.H. acknowledges Excellent Young Researcher Overseas Visit Program of JSPS that allowed him to visit LLNL (2009-2010). Computing support came from the LLNL institutional Computing Grand Challenge Program and from an INCITE Award on the Titan supercomputer of the Oak Ridge Leadership Computing Facility (OLCF) at ORNL.

\section{References}

[1] E.G. Adelberger et al., Rev. Mod. Phys. 70, 1265 (1998)

[2] E.G. Adelberger et al., Rev. Mod. Phys. 83, 195 (2011)

[3] K.M. Nollett, Phys. Rev. C 63, 054002 (2001)

[4] B.S. Nara Singh, M. Hass, Y. Nir-El, G. Haquin, Phys. Rev. Lett. 93, 262503 (2004)

[5] D. Bemmerer et al. (LUNA Collaboration), Phys. Rev. Lett. 97, 122502 (2006)

[6] T.A.D. Brown et al., Phys. Rev. C 76, 055801 (2007)

[7] F. Confortola et al., Phys. Rev. C 75, 065803 (2007)

[8] A. Di Leva et al., Phys. Rev. Lett. 102, 232502 (2009)

[9] M. Carmona-Gallardo et al., Phys. Rev. C 86, 032801 (2012)

[10] C. Bordeanu et al., Nucl. Phys. A 908, 1 (2013)

[11] S. Burzyński, K. Czerski, A. Marcinkowski, P. Zupranski, Nucl. Phys. A 473, 179 (1987)

[12] C.R. Brune, R.W. Kavanagh, C. Rolfs, Phys. Rev. C 50, 2205 (1994)

[13] T.A. Tombrello, P.D. Parker, Phys. Rev. 131, 2582 (1963)

[14] T. Kajino, Nucl. Phys. A 460, 559 (1986)

[15] T. Mertelmeier, H.M. Hofmann, Nucl. Phys. A 459, 387 (1986)

[16] A. Csótó, K. Langanke, Few-Body Syst. 29, 121 (2000)

[17] T. Neff, Phys. Rev. Lett. 106, 042502 (2011)

[18] S. Baroni, P. Navrátil, S. Quaglioni, Phys. Rev. Lett. 110, 022505 (2013); Phys. Rev. C 87, $034326(2013)$

[19] D. Baye, P. Descouvemont, Nucl. Phys. A 407, 77 (1983)

[20] P. Navrátil, J.P. Vary, B.R. Barrett, Phys. Rev. Lett. 84, 5728 (2000); Phys. Rev. C 62, 054311 (2000)

[21] S. Quaglioni, P. Navrátil, Phys. Rev. Lett. 101, 092501 (2008); Phys. Rev. C 79, 044606 (2009)

[22] D. Baye, P.-H. Heenen, M. Libert-Heinemann, Nucl. Phys. A 291, 230 (1977)

[23] P. Descouvemont, D. Baye, Rep. Prog. Phys. 73, 036301 (2010)

[24] D.R. Entem, R. Machleidt, Phys. Rev. C 68, 041001 (2003)

[25] F. Wegner, Annalen der Physik 506, 77 (1994)

[26] S.K. Bogner, R.J. Furnstahl, R.J. Perry, Phys. Rev. C 75, 061001 (2007)

[27] E.D. Jurgenson, P. Navrátil, R.J. Furnstahl, Phys. Rev. Lett. 103082501 (2009); Phys. Rev. C 83, 034301 (2011)

[28] R.J. Spiger and T.A. Tombrello. Phys. Rev. 163 (1967) 964.

[29] W.R. Boykin, S.D. Baker, and D.M. Hardy. Nucl. Phys. A 195 (1972) 241. 